\title{
Anisotropy of Magnetic Field and Velocity Fluctuations in the Solar Wind
}

\author{
Jana Šafránková ${ }^{1}$ (1) Zdeněk Němeček $^{1}$ (i) , František Němec ${ }^{1}$, Victor Montagud-Camps ${ }^{1}$ (i), Daniel Verscharen ${ }^{2,3}$ (1), \\ Andrea Verdini ${ }^{4}\left(\right.$ D) , and Tereza Ďurovcová ${ }^{1}$ \\ ${ }^{1}$ Charles University, Faculty of Mathematics and Physics, V Holešovičkách 2, 18000 Prague 8, Czech Republic; jana.safrankova@mff.cuni.cz \\ ${ }^{2}$ Mullard Space Science Laboratory, University College London, Dorking, RH5 6NT, UK \\ ${ }^{3}$ Space Science Center, University of New Hampshire, Durham, NH 03824, USA \\ ${ }^{4}$ Dipartimento di Fisica e Astronomia, Universita di Firenze, I-50019 Firenze, Italy \\ Received 2021 January 22; revised 2021 April 8; accepted 2021 April 8; published 2021 May 27
}

\begin{abstract}
We present a large statistical study of the fluctuation anisotropy in minimum variance (MV) frames of the magnetic field and solar wind velocity. We use 2, 10, 20, and 40 minute intervals of simultaneous magnetic field (the Wind spacecraft) and velocity (the Spektr-R spacecraft) observations. Our study confirms that magnetic turbulence is a composite of fluctuations varying along the mean magnetic field and those changing in the direction perpendicular to the mean field. Regardless of the length scale within the studied range of spacecraft-frame frequencies, $\approx 90 \%$ of the observed magnetic field fluctuations exhibit an MV direction aligned with the mean magnetic field, $\approx 10 \%$ of events have the MV direction perpendicular to the background field, and a negligible portion of fluctuations has no preferential direction. On the other hand, the MV direction of velocity fluctuations tends to be distributed more uniformly. An analysis of magnetic compressibility and density fluctuations suggests that the fluctuations resemble properties of Alfvénic fluctuations if the MV direction is aligned with background magnetic field whereas slow-mode-like fluctuations have the MV direction perpendicular to the background field. The proportion between Alfvénic and slow-mode-like fluctuations depends on plasma $\beta$ and length scale: the dependence on the solar wind speed is weak. We present 3D numerical MHD simulations and show that the numerical results are compatible with our experimental results.
\end{abstract}

Unified Astronomy Thesaurus concepts: Solar wind (1534); Fast solar wind (1872); Slow solar wind (1873); Heliosphere (711); Interplanetary turbulence (830); Alfven waves (23)

\section{Introduction}

The anisotropy of the solar wind and/or interplanetary magnetic field fluctuations contains important information about the solar wind origin and on the evolution of the turbulent cascade during the solar wind expansion. The wavevector anisotropy of the interplanetary magnetic field (IMF) and velocity fluctuations were for the first time observed by Belcher \& Davis (1971), and discussed extensively in the literature (e.g., Belcher \& Solodyna 1975; Burlaga \& Turner 1976; Bavassano et al. 1982; Tu et al. 1989; Marsch \& Tu 1990).

Solar wind measurements reveal the existence of two wavevector geometries of turbulent fluctuations (e.g., Matthaeus et al. 1990; Zank \& Matthaeus 1992, 1993; Tu \& Marsch 1994; Bieber et al. 1996; Podesta \& Gary 2011; He et al. 2012; Zank et al. 2017, 2020). Using abbreviations $k_{\perp}$ and $k_{\|}$for perpendicular and parallel wavevector components, the first geometry consists of oblique fluctuations with $k_{\perp}>k_{\|}$and the second geometry exhibits fluctuations that are more field aligned $\left(k_{\perp}<k_{\|}\right)$with lower amplitudes. A possible interpretation of this finding is the simultaneous coexistence of two-dimensional (2D) $\left(k_{\|} \sim 0\right)$ turbulent and slab $\left(k_{\perp} \sim 0\right)$ fluctuations (e.g., Verscharen et al. 2019; Zhao et al. 2020a). In the strict sense, purely 2D turbulence is characterized by a wavevector exactly perpendicular to the background magnetic field, $B 0$, whereas purely slab turbulence is characterized by a wavevector exactly parallel to $B 0$; however, these extreme situations are very rare in the solar wind, meaning that the power of these fluctuations is distributed across a finite range of angles around the 2D and slab limits.
Dasso et al. (2005) investigated the correlation anisotropy of solar wind MHD fluctuations and showed that the nature of the anisotropy differs in fast $\left(>500 \mathrm{~km} \mathrm{~s}^{-1}\right)$ and slow $\left(<400 \mathrm{~km} \mathrm{~s}^{-1}\right)$ winds. Fast streams ("younger" solar wind) have a larger population of fluctuations with wavevectors quasiparallel to the local magnetic field (slab geometry), while turbulence in slow ("older") streams appears to be more evolved and dominated by quasi-2D fluctuations (see also (Marsch \& Tu 1990).

Theoretically and observationally, it was found that the ratio between 2D and slab turbulence energies is 80:20 (Zank \& Matthaeus 1992; Bieber et al. 1996). A comprehensive review of observational results of slab $+2 \mathrm{D}$ fits to magnetic spectra in the inertial range was presented by Oughton et al. (2015) in the slow $\left(<400 \mathrm{~km} \mathrm{~s}^{-1}\right)$ and fast $\left(>500 \mathrm{~km} \mathrm{~s}^{-1}\right)$ winds. Observations performed in a broad range of distances from the Sun $(0.3-5 \mathrm{au})$ show the 2D fraction ranging from $50 \%-100 \%$ in the fast wind with no clear dependence on the distance in the inertial range of frequencies. The portion of $2 \mathrm{D}$ fluctuations is even larger in the slow wind. By contrast, this portion does not exceed $35 \%(16 \%)$ in the dissipation range of the fast (slow) wind. An exception was found in corotating interaction regions (CIRs) where 2D fluctuations strongly dominate (80\%-100\%) in the fast wind but they are not so abundant in the slow wind in front of CIRs. This ratio can change not only with the solar wind speed but also depending on whether measurements are performed at solar maximum or minimum, in low- or high- $\beta$ environments, and at higher or lower latitudes (Adhikari et al. 2017; Zank et al. 2017).

On the other hand, the quasi-2D turbulence is dominated by the coherent structures embedded in the solar wind like 
magnetic islands or flux ropes (Zank et al. 2017). A dependence of the ratio between $2 \mathrm{D}$ and slab turbulence on the distance from the Sun can be derived from a comparison of measurements near the Sun (Zhao et al. 2020b) by the Parker Solar Probe and at $5 \mathrm{au}$ by Ulysses (Zhao et al. 2018b). Moreover, it should be noted that the prevalence of Alfvénic structures significantly suppresses a detectability of such structures at closer heliospheric distances (Chen et al. 2020).

As an alternative to the slab+2D wavevector anisotropy model, Saur \& Bieber (1999) showed that solar wind measurements are also compatible with a combination of 2D and radial-slab (wavevectors mostly aligned with the radial direction) geometries, with a proportion of $64 \%$ for the $2 \mathrm{D}$ and $36 \%$ for the radial-slab components. The existence of such a composite anisotropy at large scales has been studied and supported in recent years with numerical results from expanding box MHD simulations (Verdini \& Grappin 2016; Montagud-Camps et al. 2018, 2020).

The direction and strength of the mean background magnetic field are important factors for the development of plasma turbulence. They affect the distribution of wavevectors in Fourier space and the autocorrelations in spatial-increment space. The background field also determines turbulence anisotropy in the sense of a component or variance anisotropy, which describes the dependence of the fluctuation power on the angle with respect to the magnetic field direction. A useful method for the analysis of this anisotropy is the minimum variance analysis (MVA), for which the fluctuations are rotated into the minimum variance (MV) reference frame (Bruno \& Carbone 2013). This is a reference frame with one of its axes aligned with the direction along which the field has the smallest fluctuation amplitude (Sonnerup \& Cahill 1967; Klein et al. 1993). Zhao et al. (2018a) have discussed in details the MVA method in order to find the background magnetic field direction at $1 \mathrm{au}$. They found that the MV magnetic field direction is basically aligned with the mean magnetic field. Considering a variety of initial MHD states, Ghosh \& Roberts (2012) investigated these changes of the MV direction with respect to the mean magnetic field and found that initial states starting with slab modes and sheared velocity streams gave the MV direction aligned with a slowly rotating background magnetic field. States with randomized noise or with slab modes and 2D structures also show a reasonable level of alignment between the MV and background magnetic field directions. These results illustrate that there is not a straightforward relation between the variance anisotropy and wavevector anisotropy.

Using the MV technique and Helios 2 data, Klein et al. (1993) examined the anisotropy of small-scale fluctuations occurring in the solar wind at a time when high- and low-speed streams were clearly distinguished. They found that variance directions of velocity fluctuations are generally aligned with the mean magnetic field in high-speed and relatively low- $\beta$ streams (where $\beta$ is the ratio between thermal and magnetic pressures). They are more isotropic in low-speed, high- $\beta$ intervals.

At large scales $\left(k \rho_{i} \gg 1, \rho_{i}\right.$ being ion gyroradius), turbulent fluctuations share properties with the three MHD wave modes in a collisionless plasma: the Alfvén wave and the fast and slow magnetosonic waves (Tu \& Marsch 1995; Klein et al. 2014), all of them having kinetic counterparts at smaller scales with properties similar to the MHD modes (Klein et al. 2012). Turbulence in the solar wind is mainly Alfvénic and thus noncompressive. The minor compressive energy in the inertial range constitutes $10 \%$ or less of the total turbulent energy and consists of slow-wave-like fluctuations, with a negligible contribution from fast waves (Howes et al. 2012; Klein et al. 2012). The compressive portion increases in the kinetic range due to the excitation of kinetic modes (e.g., Šafránková et al. 2019).

Bruno \& Bavassano (1993) analyzed the loss of the Alfvénic character of the MHD fluctuations in the inner heliosphere over time intervals with durations from 2-10 hr. Their study underlined that the dynamical interaction of these fluctuations with quasi-2D nonlinear coherent structures like magnetic islands, flux ropes, or vortexes embedded in the wind (Zhao et al. 2018b, 2020b) plays an important role in reducing the Alfvénic character of the fluctuations. Decoupling phenomena like parametric decay with a consequent direct energy cascade toward dissipative small scales (Tu et al. 1989), compressive waves, convected pressure imbalances, or other density and/or field variations (Klein et al. 1993) potentially contribute to this effect.

The present paper studies the variance anisotropy of the magnetic field and solar wind velocity and discusses their changes with plasma $\beta$ using fast Spektr-R measurements of the solar wind velocity and combining them with fast IMF measurements by Wind for the determination of the respective MV frames (Sonnerup \& Cahill 1967). The frames as well as mean magnetic field and solar wind velocity directions are computed over intervals of variable length to estimate the evolution of the turbulence anisotropy as the fluctuations cascade to smaller scales. We use 3D MHD numerical simulations to evaluate the influence of several plasma parameters on the angle between the MV direction and the mean magnetic field. By contrast to previous studies that discuss proportions among different types of fluctuations coexisting in space and time, our analysis shows that the solar wind at $1 \mathrm{au}$ is predominantly Alfvénic with randomly distributed "spots" occupied by more compressive fluctuations.

\section{Analysis of Experimental Data}

The BMSW instrument onboard Spektr-R (Šafránková et al. 2013) provides the solar wind parameters with a time resolution of $\approx 32 \mathrm{~ms}$ between the 2011 and $2017 \mathrm{yr}$. The analysis described in Sections $2.1-2.3$ is performed on $\approx 35,000$ partly overlapping 20 minute subintervals of velocity measurements (Šafránková et al. 2015). First, we identify intervals of continuous solar wind observations outside the foreshock. Then, the power spectrum in a particular solar wind interval was calculated over the first 20 minutes. The time span for the calculation of the next spectrum was shifted by 1 minute, and thus two consecutive spectra are overlapped by 19 minutes. Section 2.4 uses different durations of subintervals with the same overlapping scheme. The Spektr-R magnetometer is not in operation, thus we propagate high-time resolution magnetic field data with a cadence of $10 \mathrm{~Hz}$ from Wind (Lepping et al. 1995) for selected velocity intervals. Since Wind and Spektr-R are not necessarily in the same solar wind stream, we analyze only those intervals in which the difference between the mean solar wind speeds determined by both spacecraft is less than $5 \%$. The time of propagation of the magnetic field between Wind and Spektr-R locations was computed as a two-step approximation from Wind solar wind velocity measurements: (i) we suppose the solar wind velocity equal to $400 \mathrm{~km} \mathrm{~s}^{-1}$ and determine the time lag from the difference of spacecraft 


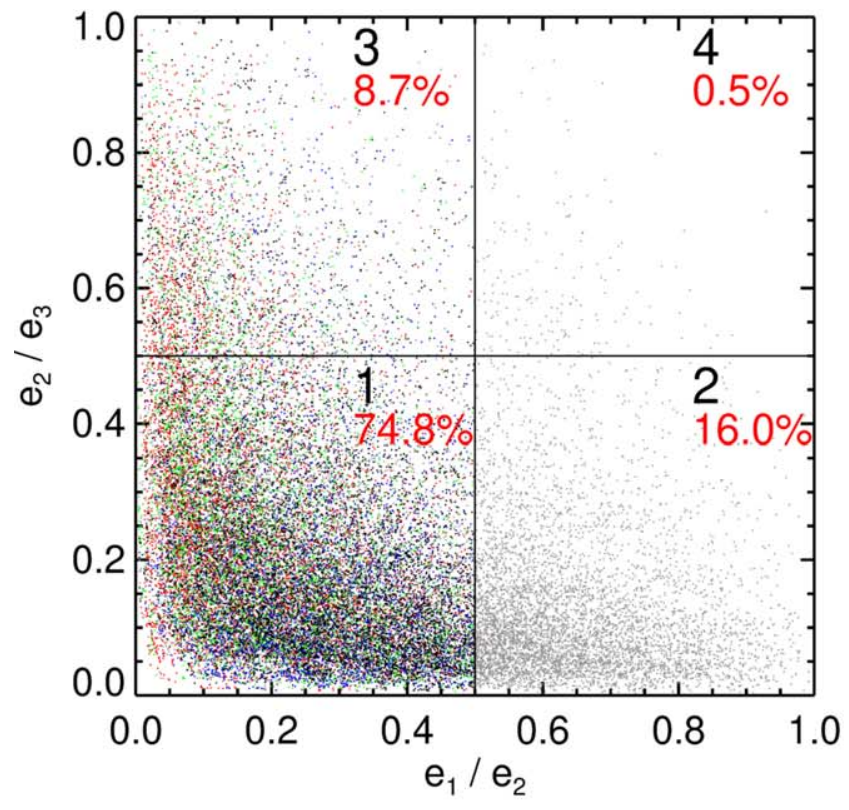

(a)

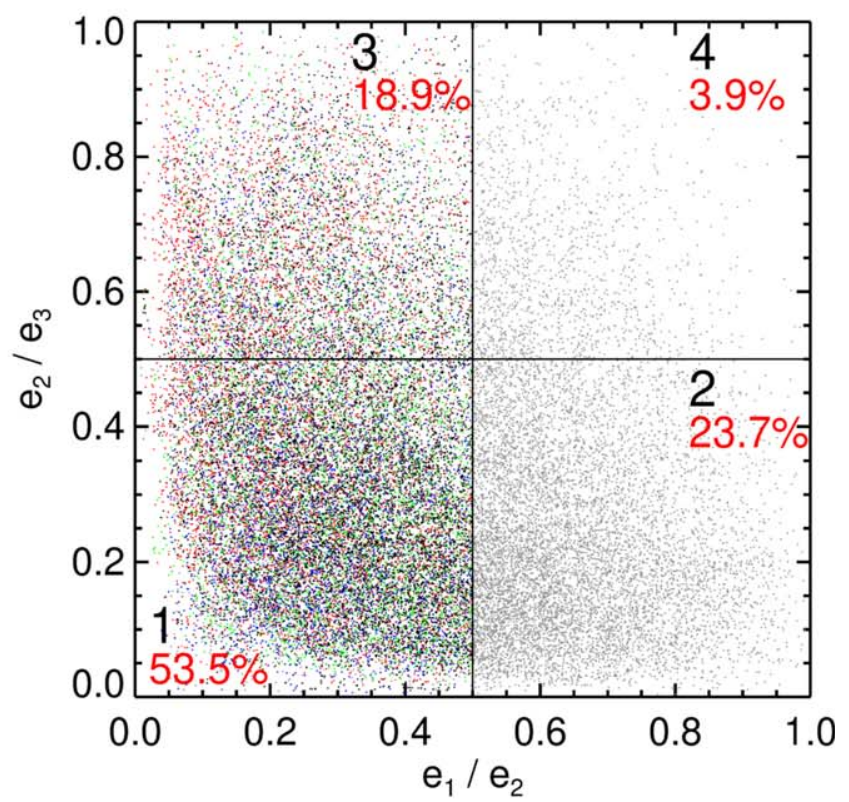

(b)

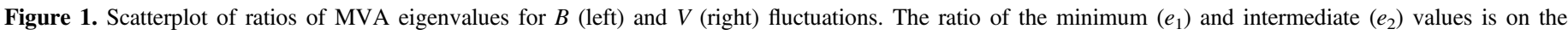

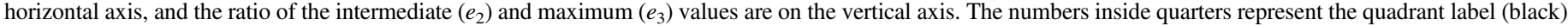

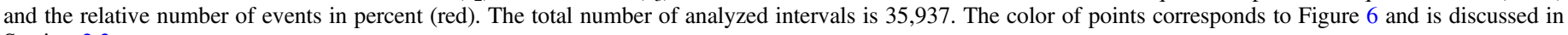
Section 2.3.

locations along the $\mathrm{X}_{\mathrm{GSE}}$ axis; (ii) then we take the actual velocity measured at the lagged time and compute the new lag (more on this procedure in Safránková et al. 2002).

\subsection{Overview of Power Anisotropy of Fluctuations}

The MVA method results in eigenvalues $e_{i}(i=1,2,3)$ that represent fluctuation power along the axis $i$. In our notation, $e_{1}$ is minimum, $e_{2}$ intermediate, and $e_{3}$ maximum eigenvalue. Based on ratios of MVA eigenvalues, $e_{i}$, we divide the analyzed intervals into four groups numbered in Figure 1. The left-hand panels show results for the magnetic field fluctuations and the right-hand panels present results for velocity fluctuations. In both panels, the left-hand quadrants $(1,3)$ have well defined MV directions $\left(e_{1} / e_{2}<0.5\right)$ whereas the minimum and intermediate values are comparable $\left(e_{1} / e_{2}>0.5\right)$ in the righthand groups $(2,4)$. Since the eigenvalues correspond to the fluctuation power in a given direction, the power isocontours in the first two quadrants have a cigar-like shape with the major axis perpendicular to the MV direction, whereas those in the third quadrant resemble a disk-like shape with the minor axis along the MV direction. The fluctuations in the bottom two quadrants $(1,2)$ can be likely identified as linearly polarized fluctuations, while those in the third quadrant are elliptically or circularly polarized. The fourth quadrant contains events characterized by quasi-isotropic fluctuations from the point of view of variance anisotropy. The prevalence of linearly polarized fluctuations (quadrants 1 and 2) is clearly seen for both quantities; though it is not so strong for velocity fluctuations, as shown by a larger portion of the intervals occupying quadrant 3 (about 9\% for the magnetic field compared to $19 \%$ for velocity variations). The portion of quasi-isotropic fluctuations (quadrant 4) is negligible for the magnetic field fluctuations but is as large as $3.9 \%$ for the velocity fluctuations.

\subsection{Directions}

This section analyzes the angles between the mean magnetic field, $B 0$, the mean bulk velocity, $V 0$, the MV direction of the magnetic field, $\mathrm{MV}_{B}$, and the $\mathrm{MV}$ direction of the velocity, $\mathrm{MV}_{V}$. We use the following notation: $\Theta_{B-B 0}$ is the angle between $\mathrm{MV}_{B}$ and $B 0, \Theta_{V-V 0}$ is the angle between $\mathrm{MV}_{\mathrm{V}}$, and $V 0, \Theta_{V-B 0}$ is the angle between $\mathrm{MV}_{V}$ and $B 0$, etc.

The MV direction is determined by the dominant fluctuations in each analyzed time interval. Thus the relation of the MV directions to the averaged $B 0$ and $V 0$ directions, scale length, and plasma parameters are of special interest. We should note that we use the same $B 0$ for the data measured by two largely separated spacecraft but Šafránková et al. (2019) have shown that the mean magnetic field, $B 0$, can be propagated to the Spektr-R spacecraft with an accuracy sufficient for statistical studies. We analyze events with low $(<0.1)$ and high $(>0.5) \beta$ separately in the following figures. The thresholds are rather close to each other but this choice provides us with a sufficient $(\approx 8000)$ number of events in both groups.

The top-left panel in Figure 2 shows the distribution of the $\Theta_{B-B 0}$ angle for both $\beta$ ranges. We find a strong preference for angles close to zero and $90^{\circ}$ in both $\beta$ ranges, although this preference is larger at low $\beta$. This preference is consistent with a combination of Alfvénic and slow-mode-like fluctuations as we show in Section 2.3. The histogram of occurrence of $\Theta_{B-V 0}$ angles in the high- $\beta$ range (top right panel, red histogram) exhibits two peaks at about $45^{\circ}$ and $135^{\circ}$. Combining the key findings in the left-hand plot (peaks close to zero and $90^{\circ}$ ) with the peaks in the right-hand panel, we interpret the distribution of angles as a consequence of fluctuations being organized according to the IMF direction with a dominant orientation along the Parker spiral. On the other hand, the low- $\beta$ events (blue histogram) exhibit only one peak at $90^{\circ}$. A distribution of random mutual orientations of the vectors in $3 \mathrm{D}$ space is 


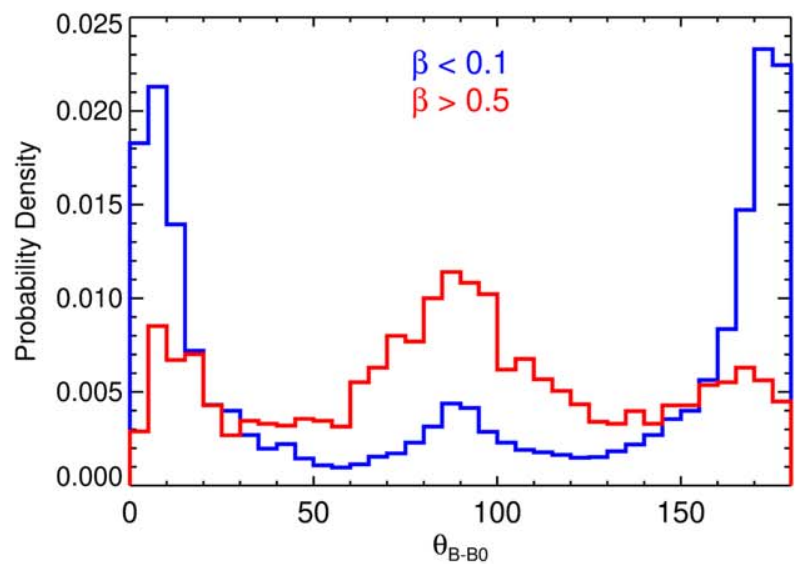

(a)

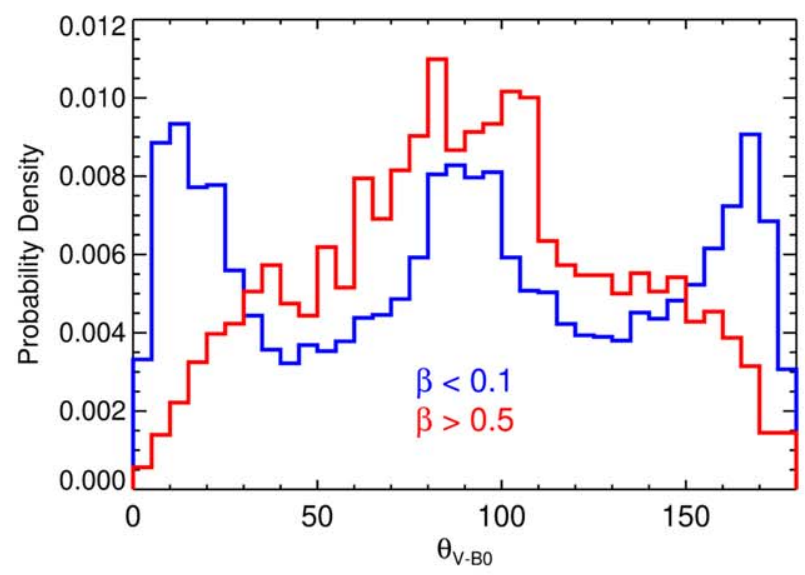

(c)

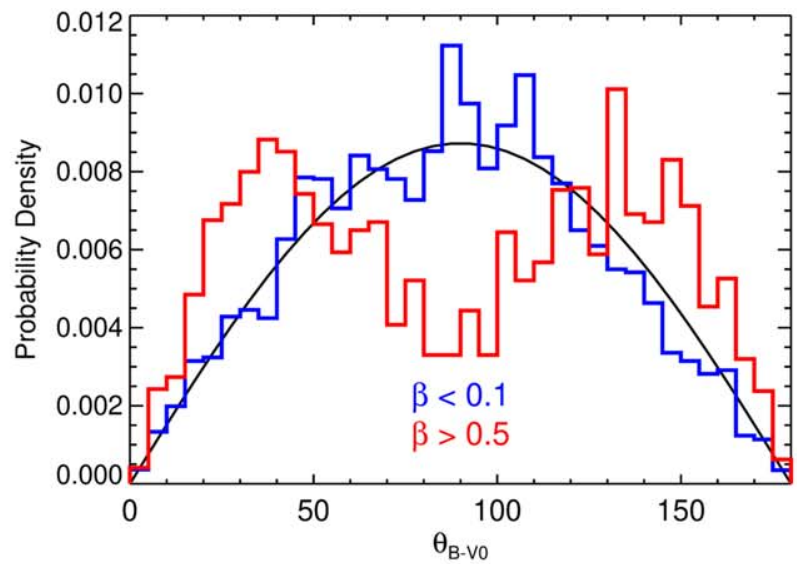

(b)

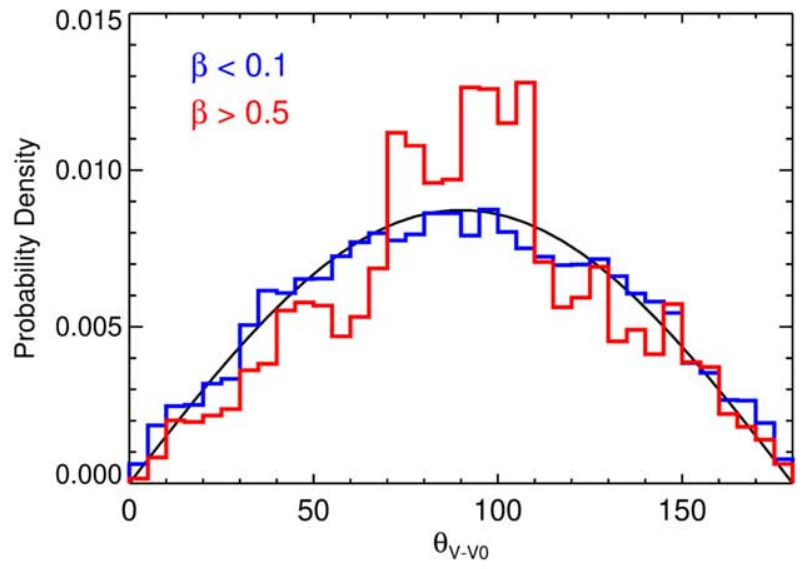

(d)

Figure 2. Top panels-distributions of angles between the $\mathrm{MV}_{B}$ direction and background magnetic field, $\Theta_{B-B 0}$ (left) and mean solar wind bulk velocity, $\Theta_{B-V 0}$ (right). Bottom panels-distributions of angles between the $\mathrm{MV}_{V}$ direction and background magnetic field, $\Theta_{B-V 0}$ (left) and mean solar wind bulk velocity, $\Theta_{V-V 0}$ (right). In both right panels, black color curves correspond to random distributions.

proportional to $\sin \Theta_{B-V 0}$, which is depicted in the right-hand panel by the black curve. The observed probability distribution of velocity fluctuations at low $\beta$ thus suggests that the distribution of $\Theta_{B-V 0}$ angles is random for low $\beta$. As can be seen by a comparison of the top and bottom panels, the velocity fluctuations tend to be more isotropic than the magnetic field fluctuations because the distributions of $\Theta_{V-B 0}$ angles (Figure 2c) are flatter or nearly isotropic than the distributions of $\Theta_{B-B 0}$ angles Figure 2(a). The distributions of the $\Theta_{V-V 0}$ angle Figure 2(d) are about random regardless of $\beta$, maybe with a slight preference for a perpendicular orientation in the high- $\beta$ environment. We think that the low background magnetic field is not able to control the direction of velocity fluctuations at high $\beta$ and preference of the perpendicular orientation is connected with the solar wind expansion. However, this preference is within the range of the statistical error.

Our analysis of the MV directions with respect to the velocity and magnetic field directions suggests that the expected IMF orientation along the Parker spiral direction is held in the high- $\beta$ but not in the low- $\beta$ wind. Figure 3 confirms this finding by showing the distribution of the IMF cone angle (the angle between the mean velocity and mean IMF directions) collected over $11 \mathrm{yr}$ of Wind observations. Also this distribution is random in the low- $\beta$ wind. The investigation of factors leading to this $\beta$ dependence is beyond the scope of the present paper and we will address it in a follow-up study.

For the sake of completeness, Figure 4 shows the distribution of angles $\Theta_{B-V}$ between MV directions of the magnetic field and velocity. The figure demonstrates that these two directions are parallel more frequently in a low- $\beta$ environment whereas the distribution of $\Theta_{B-V}$ is random for larger $\beta$. As already noted, our MVA of the velocity uses data from Spektr-R and our MVA of the magnetic field is calculated using Wind data shifted by the ballistic propagation to the Spektr-R location. Therefore, we cannot exclude a rotation of the $\mathrm{MV}_{B}$ direction along the path between two spacecraft.

\subsection{Relation between $M V_{B}$ and $M V_{V}$ Directions in $B 0$ Frame}

The analysis in the previous section has shown the importance of the $B 0$ direction for the fluctuation anisotropy. This section now analyzes the mutual relation between $\Theta_{B-B 0}$ and $\Theta_{V-B 0}$. Since the MV direction is not always well defined, we use only intervals with $e_{1} / e_{2}<0.5$ for both quantities (quadrants 1 and 3 in Figure 1). This condition reduces the number of analyzed intervals from about 36,000 to about 22,000 .

The distribution of events in $\Theta_{B-B 0} / \Theta_{V-B 0}$ space is shown in the left panel of Figure 5. The plot is symmetric in both 


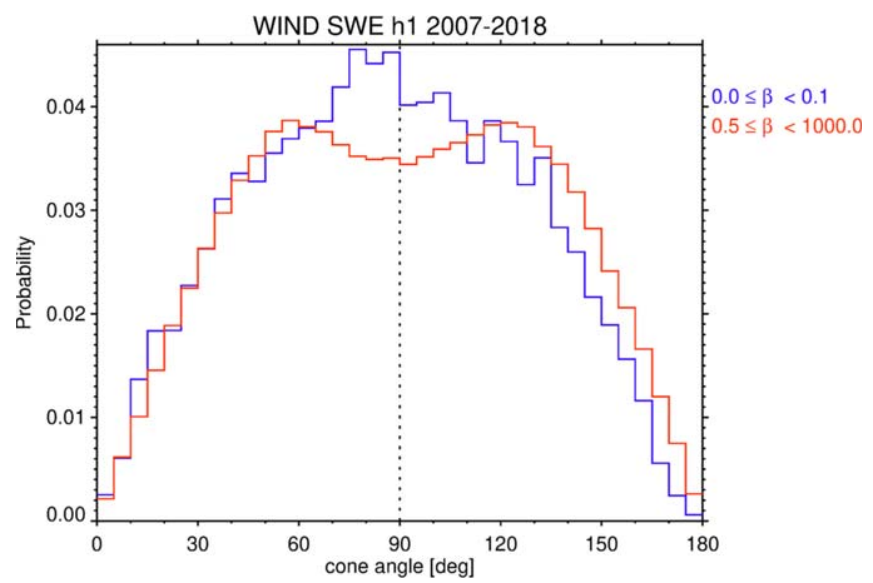

Figure 3. Distributions of angles between the mean magnetic field, $B 0$, and the mean solar wind velocity, $V 0$, in low (blue) and high (red) $\beta$ streams.

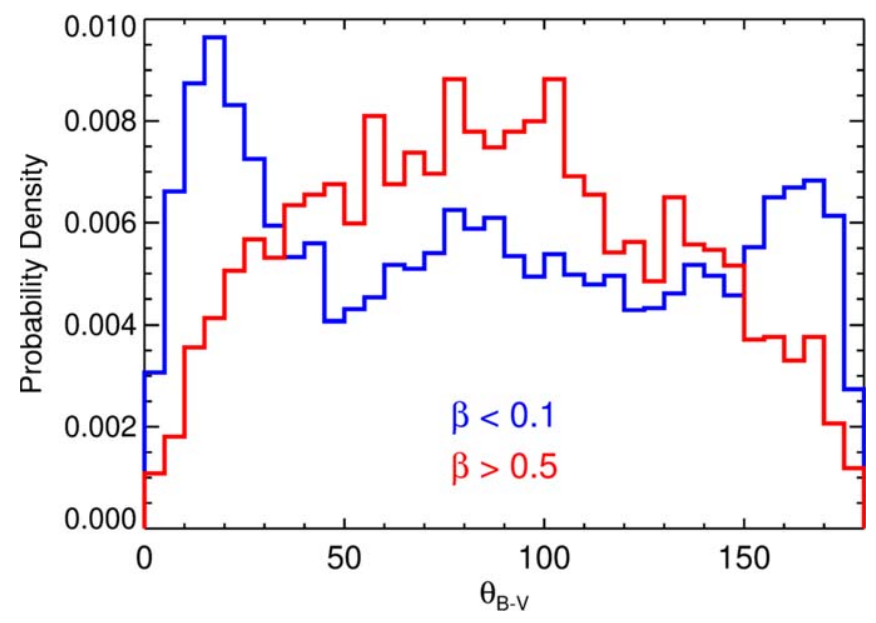

Figure 4. Distributions of angles, $\Theta_{B-V}$, between $\mathrm{MV}_{B}$ and $\mathrm{MV}_{V}$.

horizontal and vertical directions because the MVA does not resolve the ambiguity in the orientation of the MV vectors. The events are concentrated close to $\Theta_{B-B 0}=0^{\circ}$ and $180^{\circ}$ with an exception of the area indicated by the blue square where both $\Theta_{B-B 0}$ and $\Theta_{V-B 0}$ are close to $90^{\circ}$. Upon closer inspection, we find a larger density of points in the area around $\Theta_{B-B 0}=0$ and $\Theta_{V-B 0}=90^{\circ}$, which is distinguished by two green rectangles.

The right-hand panel in Figure 5 shows the probability of observations of each angle combination in the $\Theta_{B-B 0} / \Theta_{V-B 0}$ plane normalized to the distribution of random directions. A value above the dark-blue background marks a statistically significant measurement. All distinguished areas in the scatterplot in the left-hand panel are thus statistically significant. This also confirms that the peak at the center of the plot on the left is not merely the result of a random distribution but a statistically significant signal.

We conjecture that the intervals in the red squares are occupied with parallel Alfvénic fluctuations and, likewise, the blue square represents slow-mode-like variations propagating perpendicular to $B 0$. Note that this analysis is not sufficient for a determination of wave modes. Our classification is based on conclusions of Šafránková et al. (2019) that the power spectral densities of magnetic field and velocity fluctuations in the inertial range can be successfully modeled by a combination of slow and Alfvénic fluctuations but we cannot exclude a presence of fast modes (Howes et al. 2012; Klein et al. 2012).
Further evidence for such interpretation is shown in Figure $6 \mathrm{~b}$. The green area is more difficult to interpret, but the classification as slow-mode-like waves propagating along $B 0$ seems to be also possible. The colored areas contain altogether about $65 \%$ of all points. We interpret the points outside the rectangles as a combination of both modes, a dominance of other wave modes like kinetic Alfvén waves (KAWs) that propagate in arbitrary directions with respect to the magnetic field, uncertainties in a determination of MV directions, or due to uncertainties following from the propagation of the mean magnetic field direction to Spektr-R.

In the previous section, we have shown that plasma $\beta$ is an important factor influencing the MV directions. Therefore, in the left panel of Figure 6, we plot the distributions of this parameter for the regions distinguished in Figure 5. Nearly all analyzed events have $0.03<\beta<3$. Nevertheless, we observe a clear difference between the distributions that we interpret as Alfvénic fluctuations (red histogram) and those attributed to slow-mode-like variations (blue histogram). The distribution for the events classified as slow-mode-like fluctuations peaks at $\beta \approx 0.4$ whereas the distribution corresponding to Alfvénic fluctuations is shifted toward low $\beta$ with a peak around $\beta \approx 0.2$.

The classification of fluctuations as Alfvénic in the red area and slow-mode-like in the blue area is supported by the right panel of Figure 6. The scatterplots of the density, $\mathrm{d} n / n$, and magnetic field magnitude $\mathrm{d}|B| /|B|$ relative fluctuations reveal a much larger density and/or magnetic field strength variations for the fluctuations with the MV direction perpendicular to the magnetic field (blue region). The median values of $\mathrm{d} n / n$ are 0.04 in the red and 0.08 in the blue regions, and the medians of $\mathrm{d}|B| /|B|$ are 0.02 and 0.11 , respectively. One would intuitively expect that the nature of the fluctuations in the colored areas also be connected to their linear or elliptical polarization. For this reason, Figure 1 uses the same color scheme as Figure 6 but a deeper look at the distribution of colors in Figure 1 shows no clear preference for any color to occupy a particular sector.

We discuss the anisotropy characteristics in terms of their dependence on $\beta$. Many authors attribute changes in wavevector anisotropy to variations of the solar wind speed connected with their source regions on the Sun (e.g., Marsch \& Tu 1990; Dasso et al. 2005; Oughton et al. 2015). It is of interest to verify whether the solar wind speed also affects variance anisotropy as it does wavevector anisotropy. For this reason, upper panels in Figure 7 show the probability map of the observation of a particular $\Theta_{B-B 0}$ and $\Theta_{V-B 0}$ combination normalized to a random distribution for subsets of events with $\beta<0.1$ (left) and $\beta>0.5$ (right). The difference between the left and right panels shows that Alfvénic fluctuations dominate in low- $\beta$ plasma whereas a mix of wave modes is observed at higher $\beta$. The low- $/$ high- $\beta$ subsets are further divided into groups with low $\left(<350 \mathrm{~km} \mathrm{~s}^{-1}\right)$ and high $\left(>450 \mathrm{~km} \mathrm{~s}^{-1}\right)$ wind speeds and the probability maps for these four groups are put below the corresponding $\beta$ panels in Figure 7. We conclude that although the anisotropy characteristics are predominantly controlled by $\beta$, an increase of the relative portion of intervals dominated by Alfvénic fluctuations in the fast wind is notable in both $\beta$ groups.

\subsection{Dependence of Fluctuation Anisotropy on Scale}

Our analysis presented so far uses a 20-minute scale, thus they cover frequencies above $0.001 \mathrm{~Hz}$ in the spacecraft frame, which is well inside the MHD regime. In order to check the 


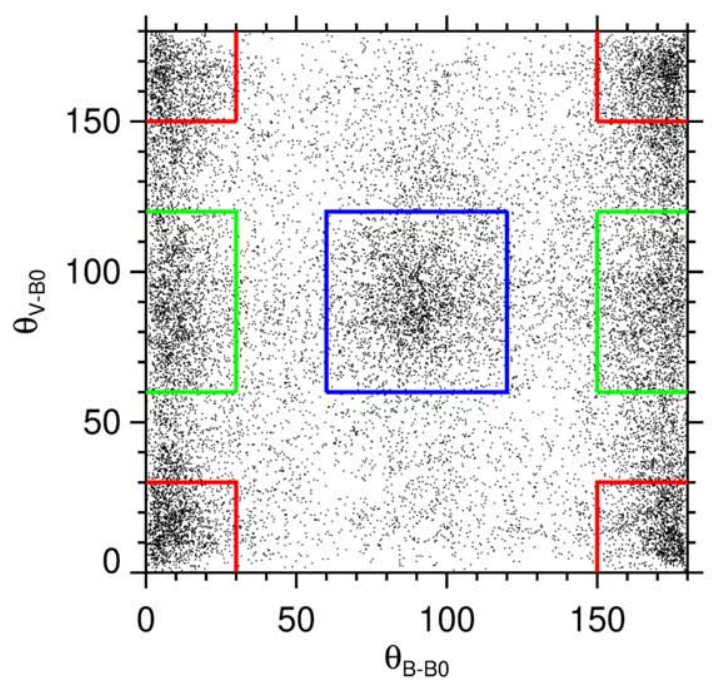

(a)
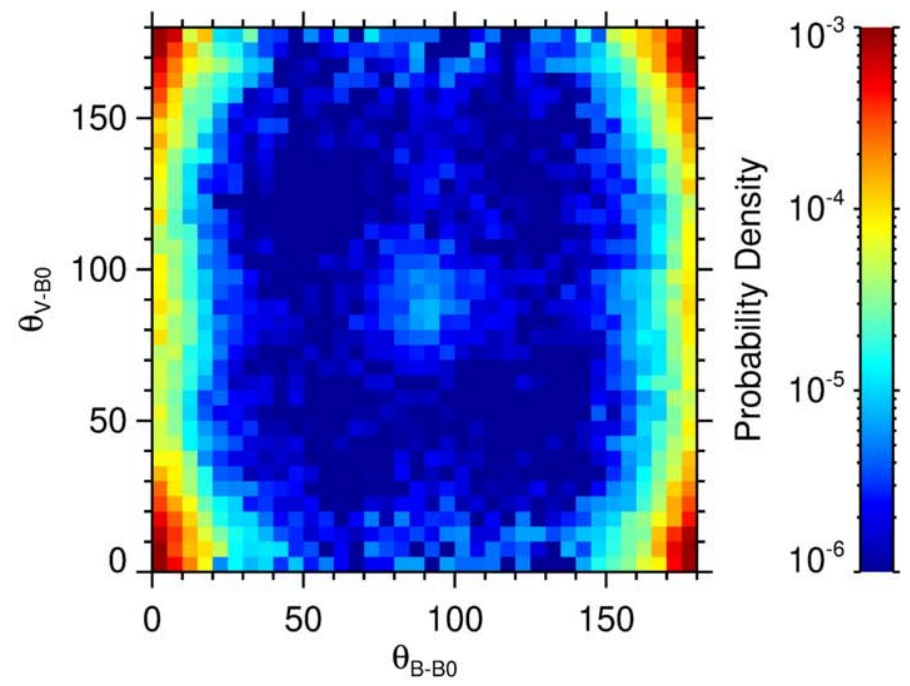

(b)

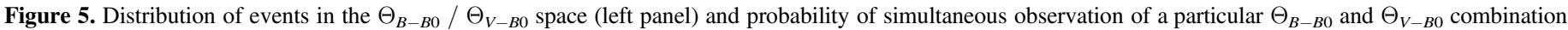
normalized to a random distribution (right panel).

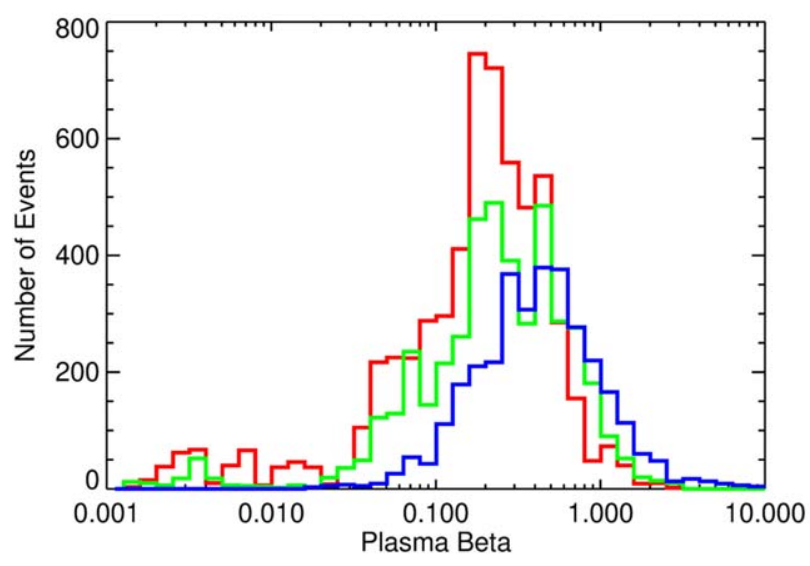

(a)

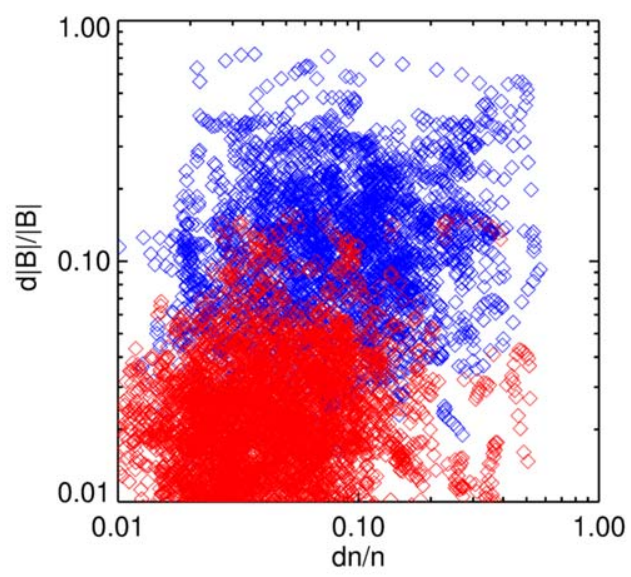

(b)

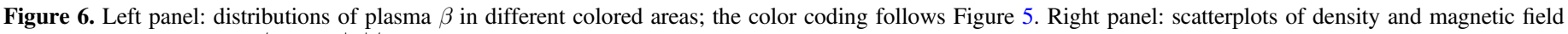
magnitude fluctuations, $\mathrm{d} n / n$ and $\mathrm{d}|B| / B$, respectively, for the events at the red and blue areas in Figure 5 .

dependence of our results on scale, we carry out our MVA on intervals with different durations. The results shown in Figure 8 suggest that the portion of the slow-mode-like interval decreases with decreasing interval length because the relative number of events in the blue rectangle increases with scale. We also compute the probability normalized to the probability of the random orientation (not shown), which again confirms the statistical significance of our results. We present statistics of the events from Figure 8 in Table 1.

The top boxes in the "Total No." column contain the total number of analyzed intervals, and the number of intervals with well determined MV directions are below. Their ratios are about constant $(\approx 0.65)$ and do not depend on the interval length. Also the analysis like the one shown in Figure 1 provides qualitatively the same results for all investigated scales. Since the total duration of the analyzed observations is limited, the number of intervals decreases with increasing interval length and thus we discuss the relative numbers given in percent in the columns denoted as "Red," "Green," and
"Blue". These colors correspond to the groups distinguished in Figures 5 and 8.

The Alfvénic fluctuations strongly dominate in 2 minute intervals and the probability of observations of slow-mode-like fluctuations (blue box) is larger in the longer intervals. This finding is consistent with the analysis of the polarization properties of turbulence by Šafránková et al. (2019). The authors have shown statistically that the compressibility of velocity fluctuations decreases with scale through the MHD range in the low- $\beta$ environment. This can be interpreted as an increasing portion of intervals dominated by Alfvénic fluctuations at shorter scales. This effect is also consistent with the loss of the Alfvénic character of magnetic field fluctuations on large (several hr) scales in the inner heliosphere noted by Bruno \& Bavassano (1993).

In order to search for differences between the local and global mean field directions (see discussion in Oughton et al. 2015), we also compute the mean magnetic field as the average over 40 minute intervals with shorter intervals in the center. However, applications of this "global" magnetic field for 


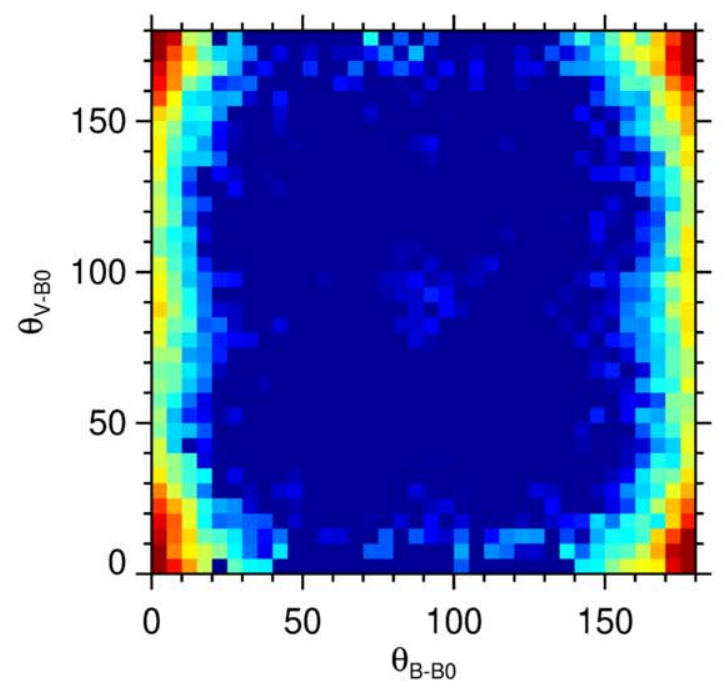

(a)
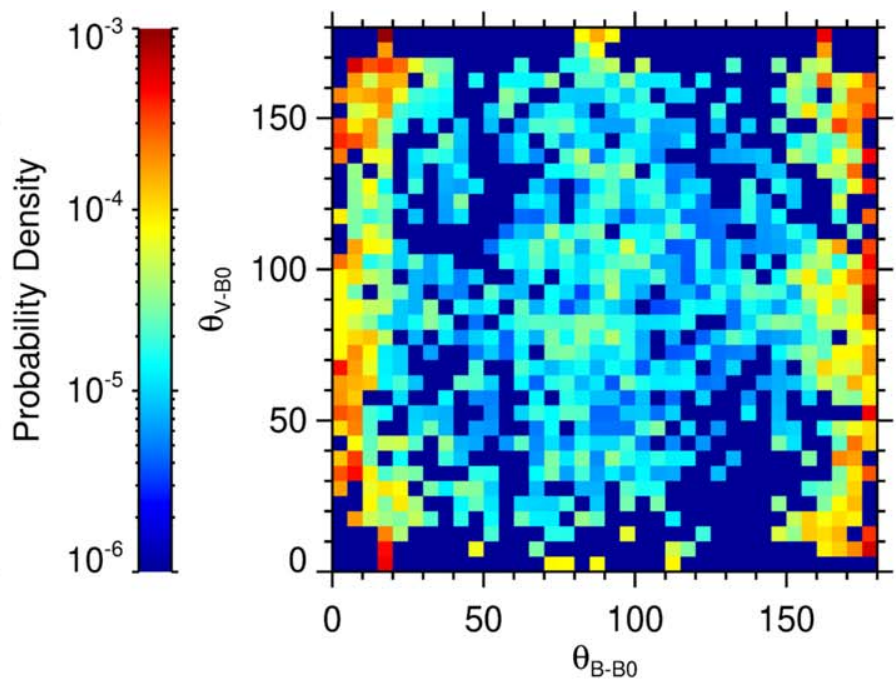

(b)

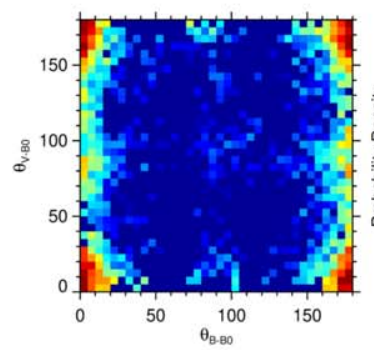

(c)
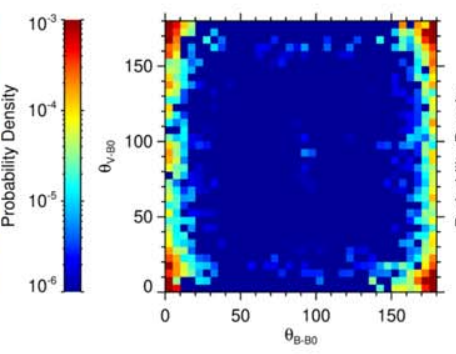

(d)
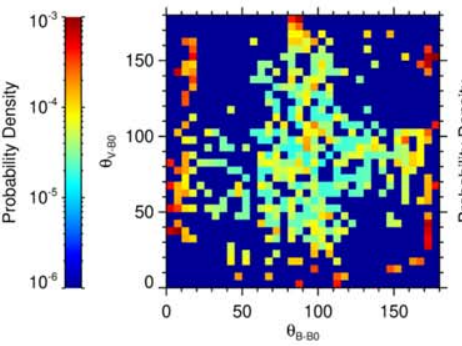

(e)
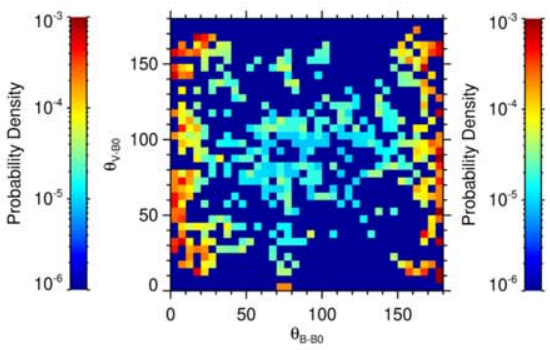

(f)

Figure 7. Top panels: normalized distributions of probability of simultaneous observations of a particular $\Theta_{B-B 0}$ and $\Theta_{V-B 0}$ combination for low- $(<0.1$, left) and high- ( $>0.5$, right) $\beta$ events; bottom panels: events from top panels sorted according to the solar wind bulk speed, $V 0<350 \mathrm{~km} \mathrm{~s}^{-1}$ (first and third panels), $V 0>450 \mathrm{~km} \mathrm{~s}^{-1}$ (second and fourth panels).

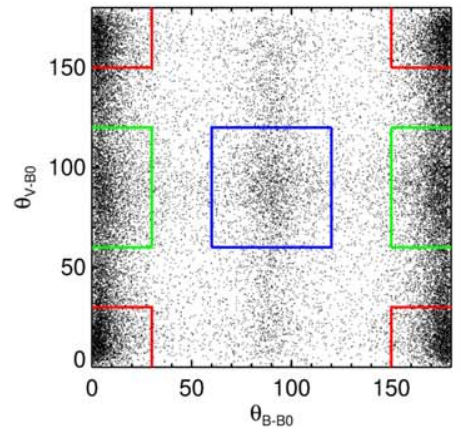

$2 \mathrm{~min}$

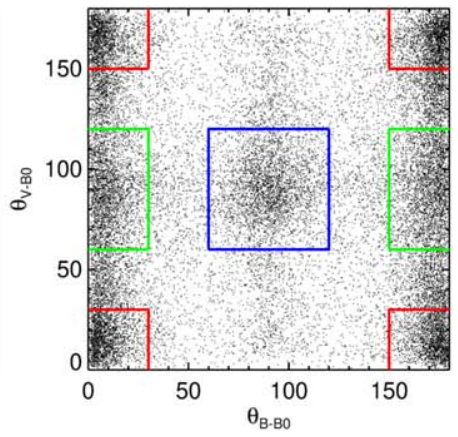

$10 \mathrm{~min}$

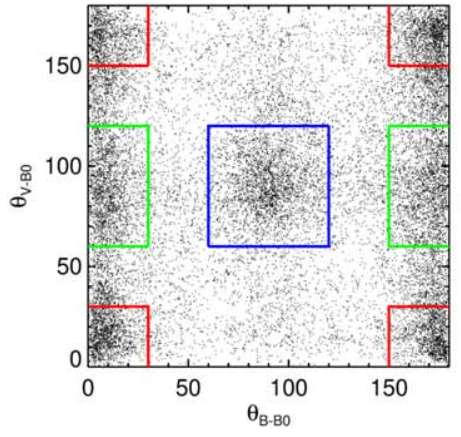

$20 \mathrm{~min}$

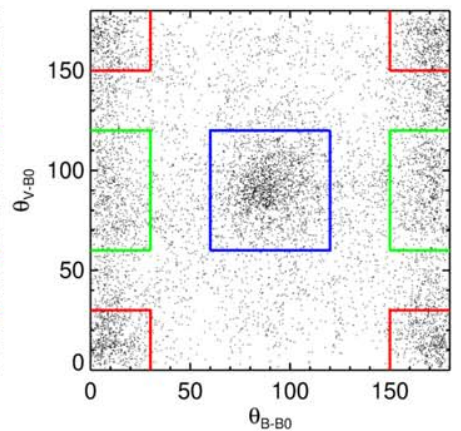

$40 \mathrm{~min}$

Figure 8. Distributions of events in the $\Theta_{B-B 0}$ and $\Theta_{V-B 0}$ space for different scales: from left to right 2, 10, 20, and 40 minutes, respectively.

Table 1

Statistics of the results from Figure 8

\begin{tabular}{lcccc}
\hline \hline Interval length & Total No. & Red & Green & Blue \\
\hline 2 minutes & 50,322 & 11,009 & 7747 & 2591 \\
& 33,360 & $33 \%$ & $23 \%$ & $7 \%$ \\
10 minutes & 45,126 & 8351 & 5937 & 3589 \\
& 28,796 & $29 \%$ & $21 \%$ & $13 \%$ \\
20 minutes & 35,937 & 5902 & 4393 & 3247 \\
& 21,859 & $27 \%$ & $20 \%$ & $15 \%$ \\
40 minutes & 16,697 & 2230 & 1578 & 2259 \\
& 9697 & $23 \%$ & $16 \%$ & $23 \%$ \\
\hline
\end{tabular}

shorter intervals provided generally the same results as those shown in Table 1; namely, a conservation of the relative number of points outside the colored areas and an increase of the portion of Alfvénic fluctuations with decreasing scale.

\section{Comparison of Observations with the MHD Model}

Previous sections have pointed out a strong relation between plasma $\beta$ and the MV angle distribution. However, plasma $\beta$ is only one of many plasma parameters that can influence the evolution of turbulence in the solar wind. It is possible that these parameters are also important for the MVA distribution but a selection of data intervals imposing conditions on 


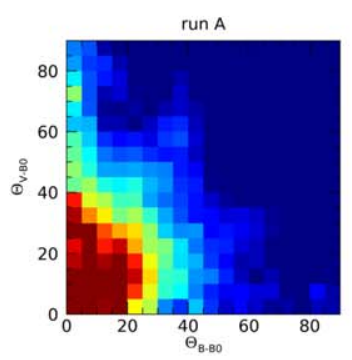

$$
\begin{gathered}
\beta=0.2 \\
\Theta_{V 0-B 0}=0^{\circ} \\
M=0.5 \\
\mathrm{~d}|B| /|B 0|=0.2
\end{gathered}
$$

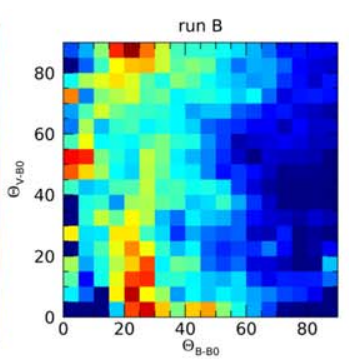

$\beta=0.2$

$\Theta_{V 0-B 0}=45^{\circ}$

$M=0.5$

$\mathrm{d}|B| /|B 0|=0.2$
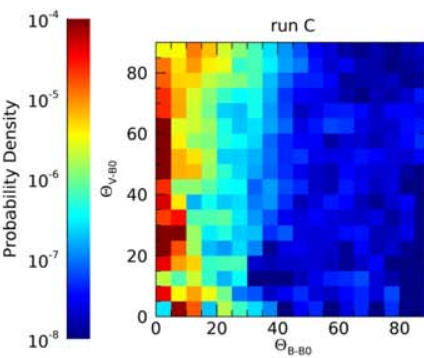

$\beta=1.0$

$\Theta_{V 0-B 0}=0^{\circ}$

$M=0.27$

$\mathrm{d}|B| /|B 0|=0.25$
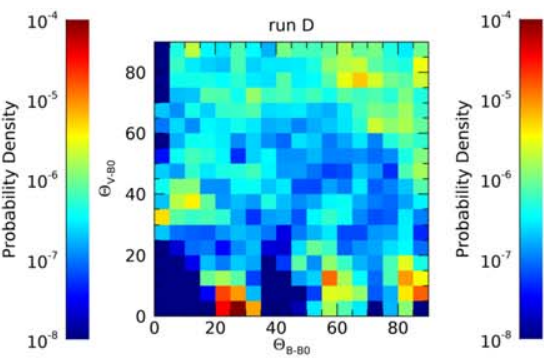

$\beta=1.0$

$\Theta_{V 0-B 0}=45^{\circ}$

$M=0.77$

$\mathrm{d}|B| /|B 0|=1$

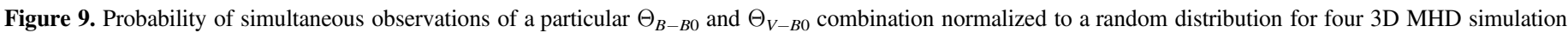
runs $\mathrm{A}, \mathrm{B}, \mathrm{C}$, and $\mathrm{D}$ (from left to right). Initial conditions are given below each panel.

multiple parameters would reduce significantly the statistics. On the other hand, numerical simulations offer the possibility to freely set the initial plasma parameters, helping therefore with the identification of those that control the MV angle distribution.

In this section, we present a series of numerical simulations with a motivation to confirm the influence of plasma $\beta$ on the MVA distribution as well as to evaluate the effect of other parameters like turbulent Mach number, $\mathrm{d}|B| /|B 0|$ ratio, and the sampling direction. Our MHD plasma model neglects the effect of mechanisms present at very large scales in the solar wind, such as expansion, and those becoming important close to kinetic scales, thus they are a suitable approximation for the behavior of the plasma in the middle of the inertial range, where the experimental observations have been made. Presented simulations use the expanding box model code (Grappin et al. 1993) without expansion and without forcing. All runs are computed on a simulation box of a size $(2 \pi)^{3}$ with a uniform grid of 256 points in each direction. We initialize our simulations with an isotropic distribution of velocity and magnetic field fluctuations, and set the initial cross helicity to zero. The dynamic viscosity, resistivity, and thermal conductivity were set to $2 \cdot 10^{-3}$ in simulation units (for details, see Montagud Camps et al. 2018) for all runs. The fluctuations are excited up to the wavenumber $k=16 / 2 \pi$ and they follow an initially isotropic spectrum proportional to $k^{-5 / 3}$. The velocity fluctuations are initially solenoidal, and the kinetic and magnetic energies are initialized at equipartition. The initial plasma parameters that we modify for the different simulation runs are: the plasma $\beta$, the turbulent Mach number, $\mathbf{M}$ ( $M=V_{\mathrm{rms}} / c_{s}$, where $c_{s}$ is the sound speed and $V_{\mathrm{rms}}$ is the initial rms velocity of the fluctuations (Montagud Camps et al. 2018)), the $\mathrm{d}|B| /|B 0|$ ratio, and the orientation of the background magnetic field with respect to the direction in which data are collected, $\Theta_{V 0-B 0}$. The background magnetic field was oriented either along the $x$ axis or declined by $45^{\circ}$ toward the $y$ axis to simulate the Parker spiral orientation.

We perform our MVA on 1D cuts of the numerical domain parallel to the $\mathrm{x}$ axis. Assuming Taylor's hypothesis, these cuts represent time intervals in observations. The time in our simulations is normalized to nonlinear time units computed at the beginning of the run $\left(t_{n l 0}=1 / k_{0} \cdot V_{\text {rms }}\right)$ and the MV angle distributions are computed at time $t=2.5 \cdot t_{n l 0}$ (note that $t_{n l 0}$ is the initial nonlinear time). For the analysis of the simulation data, we apply the same criterion for the ratio of eigenvalues, $e_{1} / e_{2}<0.5$, as in our experimental study. We discard all cuts that do not fulfill this threshold.

Since the model results depend on a number of parameters, we make several runs and compute for them distributions of MV directions. Figure 9 shows plots of the MV analysis for the runs that we later use for the comparison with our spacecraft data. Since we have only several hundreds of MV directions from one simulation, we make use of the already mentioned symmetry and mirror the angles above $90^{\circ}$ into the $0-90^{\circ}$ range to suppress noise in our plots. The color bars indicate the normalized probability. The results from runs $\mathrm{A}, \mathrm{B}$, and $\mathrm{C}$ (first three panels in Figure 9) show that turbulence with an initially low $\mathrm{d}|B| /|B 0|$ ratio favors alignments of the magnetic fluctuations with the mean magnetic field, in agreement with the observations (see Figure 6). The higher value of the initial plasma $\beta$ in run $\mathrm{C}$ compared to $\mathrm{A}$ and $\mathrm{B}$ shows the influence of this parameter on the component anisotropy of the velocity fluctuations. We should note that these runs differ also by $M$ and $\mathrm{d}|B| /|B 0|$ but examinations of about 20 runs we made under different conditions revealed $\beta$ as the most important parameter.

The difference between runs $\mathrm{A}$ and $\mathrm{B}$ shows that the angle $\Theta_{V 0-B 0}$ has a significant effect on the distribution of $\mathrm{MV}$ angles. The peak of the $\Theta_{B-B 0}$ distribution shifts from $0^{\circ}$ in run A to $15^{\circ}$ in run $\mathrm{B}$. The $\Theta_{V-B 0}$ angle exhibits one peak at $0^{\circ}$ in run $A$ and three peaks at $0^{\circ}, 45^{\circ}$, and $90^{\circ}$ in run $B$. Runs $C$ and $\mathrm{D}$ use larger initial $\beta$ and differ by the magnetic field orientation and initial $\mathrm{d}|B| /|B 0|$ ratio. The increase in $\beta$ affects predominantly the distribution of velocity fluctuations, $\Theta_{V-B 0}$ becomes random, while run $\mathrm{D}$ presents a decreased probability of the MV angles around $0^{\circ}$ for both $\Theta_{B-B 0}$ and $\Theta_{V-B 0}$. Our additional runs confirm that both plasma $\beta$ and $\mathrm{d}|B| /|B 0|$ ratio should be close to unity to obtain the large $\Theta_{V-B 0}$ angle. The peak of magnetic fluctuations shifts toward larger angles when increasing $\Theta_{V 0-\text { B0 }}$. We attribute this enhancement of the $\Theta_{V 0-B 0}$ effect observed in run D to the increase of the initial $\mathrm{d}|B| /|B 0|$ ratio up to one. Such an increase of magnetic field variations randomizes the total mean magnetic field, while the distribution of MV angles is computed with respect to this mean field.

Numerical simulations reveal that both the plasma $\beta$ and the sampling direction influence the distribution of MV angles for velocity fluctuations but the MV direction of magnetic fluctuations is more sensitive to changes of the sampling direction and $\mathrm{d}|B| /|B 0|$ ratio. In order to see a significant departure from $\Theta_{B-B 0} \approx 0$, the increase of $\beta$ should be 


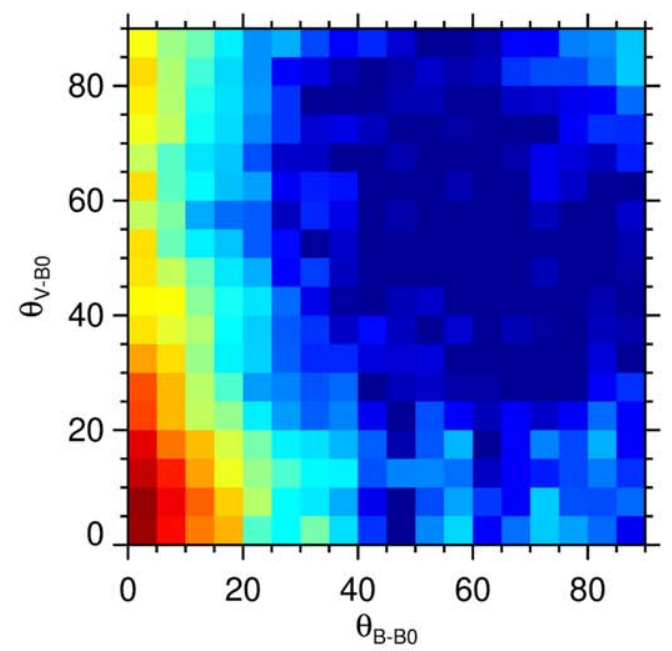

(a)

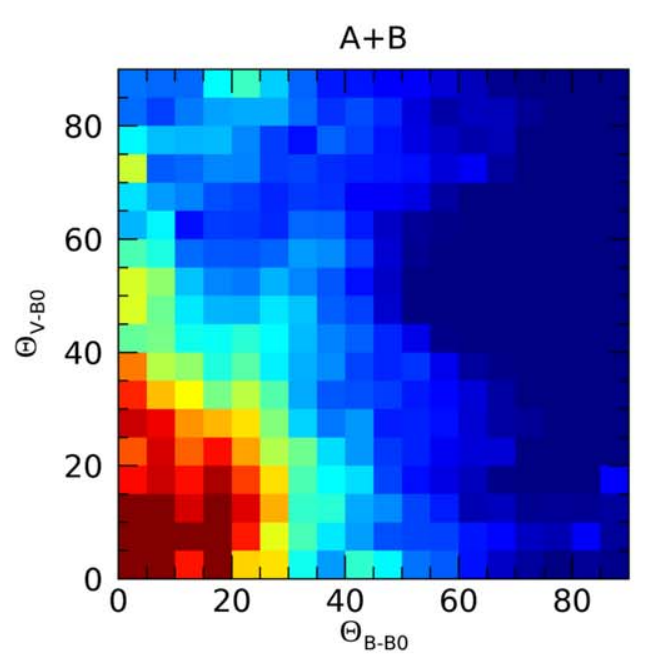

(c)

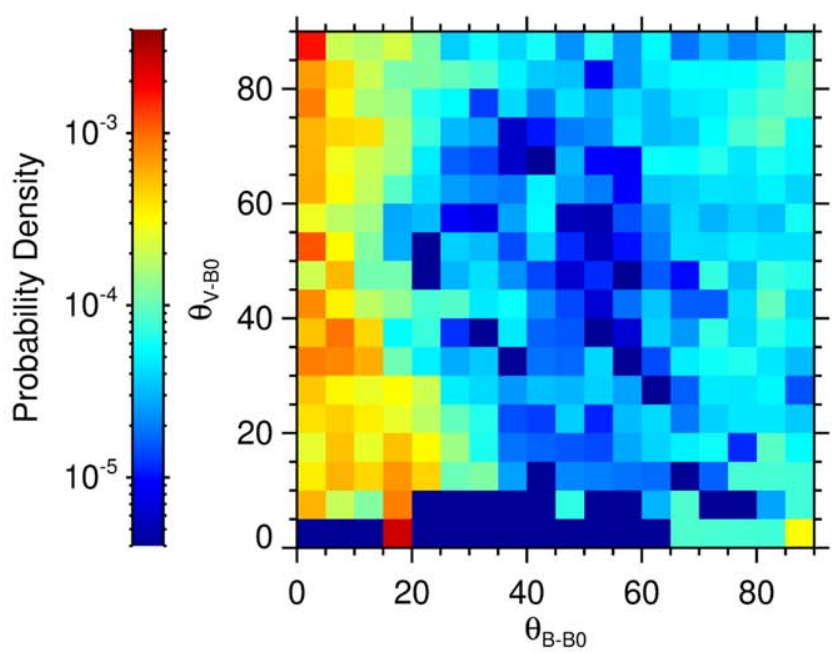

(b)
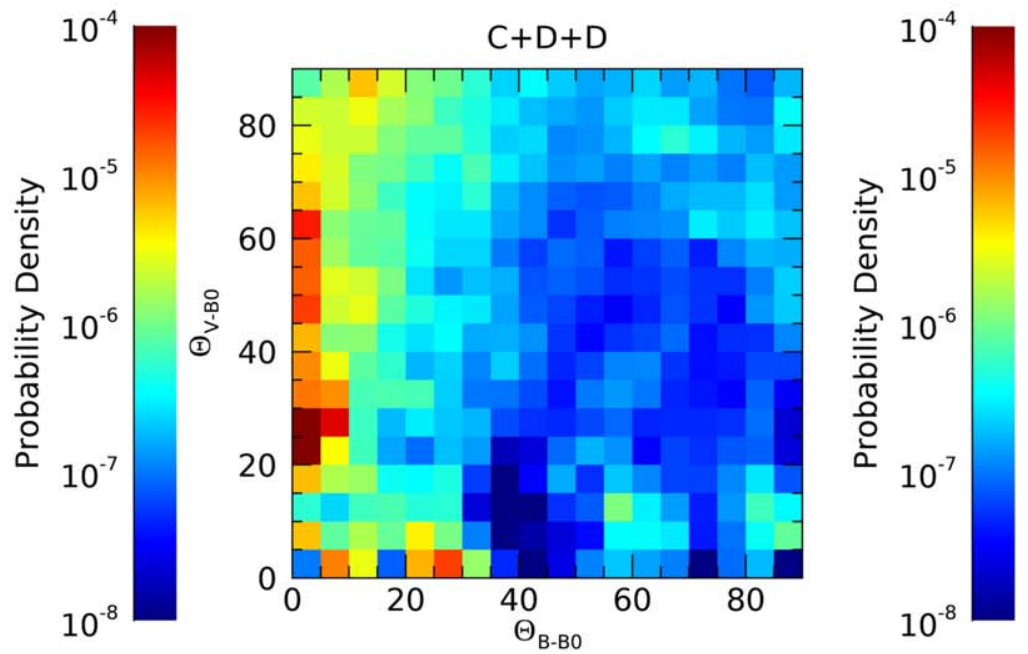

(d)

Figure 10. A comparison of experimental results with our 3D MHD model. Top panels-the probability map of $\Theta_{B-B 0} / \Theta_{V-B 0}$ angles in two ranges of plasma $\beta$ (left$\beta<0.2$, right $\beta>1$ ) from experimental data; bottom panels-simulation results for two $\beta$ cases (left $-\beta=0.2$, right $-\beta=1$ ). Other initial conditions are specified in the text and Figure 9.

complemented with a large $\mathrm{d}|B| /|B 0|$ ratio and the sampling direction around $45^{\circ}$ that is typical for the solar wind at $1 \mathrm{au}$.

None of the panels in Figure 9 resemble all features obtained in observations. For this reason, we group simulation results for the same values of plasma $\beta$ and compare them with observations in Figure 10. The top panels show the probability of observations of a particular $\Theta_{B-B 0}$ and $\Theta_{V-B 0}$ combination normalized to a random distribution for two subsets, which differ by $\beta$. The analogous plots for results of our MHD simulations are shown in the bottom panels. We have selected those simulation runs that provide a reasonable match in terms of the plasma parameters with the experimental data. The lefthand plots present low- $\beta(\leqslant 0.2)$ events. There is no principal difference between the top plot and the plot for $\beta \leqslant 0.1$ shown in Figure 7. The bottom panel corresponds to a combination of runs $\mathrm{A}$ and $\mathrm{B}$ with $\beta=0.2$ that differ by the direction of the mean magnetic field. This choice of combination reflects, to a degree, the fact that the experimental data combine all occurring values of $\Theta_{V 0-B 0}$ with a weight given by their distribution in the analyzed data set. The right-hand plots compare observed (top) and simulated (bottom) distributions of
MV directions for high $\beta(\geqslant 1)$ cases. This limit is applied to experimental data, and the bottom panel combines run $\mathrm{C}$ and two times run $\mathrm{D}(\mathrm{C}+\mathrm{D}+\mathrm{D}$ in Figure 10) to reflect the most frequent magnetic field orientation along the Parker spiral.

The results of our experimental analysis and MHD simulations are qualitatively consistent; namely, the Alfvénic fluctuations occur preferentially at smaller $\beta$, and the distribution becomes more random at larger $\beta$. In order to check whether MHD simulations also reflect the distribution of Alfvénic and slow-mode-like fluctuations, we combine the data from all runs and computed medians of $\mathrm{d} n / n$ and $\mathrm{d}|B| /|B 0|$ ratios in the red and blue areas of $\Theta_{B-B 0}-\Theta_{V 0-B 0}$ parameter space (see Figure 8) and received similar relations as those discussed in Section 2.3, namely the $\mathrm{d}|B| /|B 0|$ ratios in blue and red areas differ by a factor of 10 .

Our numerical simulations also reveal the difficulty to relate the MV angle distribution with the wavevector anisotropy. Figure 11 shows energy isosurfaces in wavevector space of the magnetic fluctuations for each simulation used in Figure 9 at the moment the MV direction is computed. Despite showing different probability distributions of the MV angles, all 


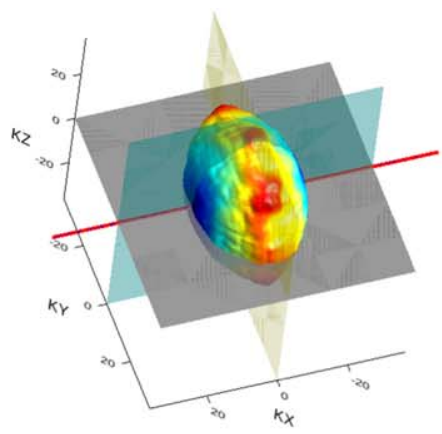

(a)

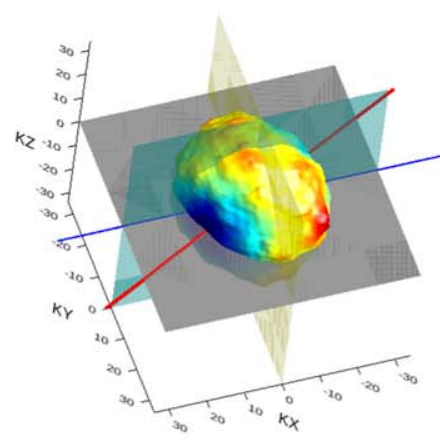

(b)

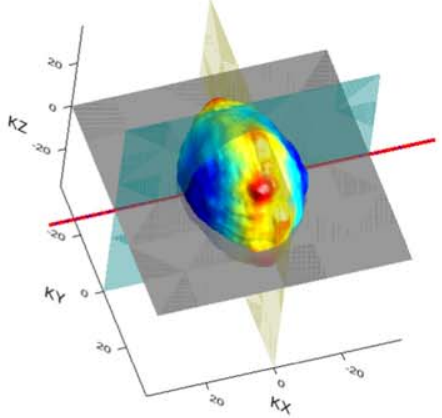

(c)

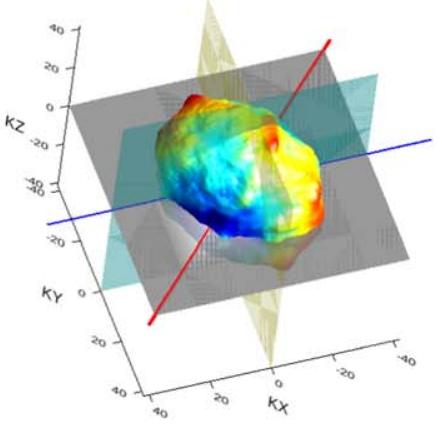

(d)

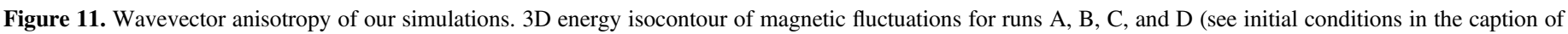

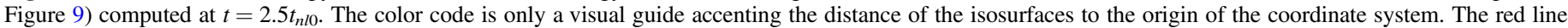

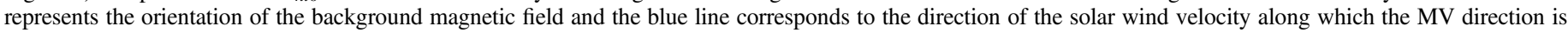
computed.

simulation runs have an oblate distribution of energy in the wavevector space with $B_{0}$ as the symmetry axis, even for run A, which exhibits a higher probability of MV angles around $90^{\circ}$ (left panel in Figure 11). This confirms that there is no direct correspondence between the MV direction probability density and the wavevector anisotropy.

Our numerical results, in accordance with Ghosh \& Roberts (2012), indicate that intervals with MV parallel or perpendicular to $B 0$ (red and blue populations in Figure 8) do not necessarily map directly to the two populations with $2 \mathrm{D}$ and slab or radial-slab wavevector anisotropies. Nevertheless, this may still be the case in the solar wind, but further work is required to understand this connection. For instance, the analysis of the data intervals from the red and blue populations in Figure 8 via magnetic and velocity autocorrelations (Matthaeus et al. 1990; Dasso et al. 2005) or via the power spectral anisotropy (Bieber et al. 1996; Hamilton et al. 2008) could give insight into their relationship with wavevector anisotropy.

\section{Summary}

Our analysis is based on $\approx 1200 \mathrm{hr}$ of Spektr-R fast measurements of the solar wind velocity vector. The same data set was used in the studies by Šafránková et al. (2015, 2016, 2019). Since the Spectr-R magnetometer is not in operation, our analysis of Spektr-R data uses the direction of the magnetic field propagated from the location of the Wind spacecraft. Šafránková et al. (2019) as well as our additional analysis confirms that this approach is applicable (although with some uncertainty) in statistical studies. We divide Spektr$\mathrm{R}$ and corresponding Wind observations into partly overlapping intervals and determine MV velocity and magnetic field directions for each of them. The MV frame is considered to be well defined if the ratios of minimum and intermediate eigenvalues of both the velocity and magnetic field are less than 0.5. We focus our analysis on the angles between the mean magnetic field $B 0$ and the MV magnetic field $\left(\Theta_{B-B 0}\right)$ or MV velocity $\left(\Theta_{V-B 0}\right)$ directions. Analogously, we use the angles between MV directions of $B$ and $V$ and the mean velocity, $V 0\left(\Theta_{V-V 0}\right.$ and $\left.\Theta_{B-V 0}\right)$. The core of our study uses 20 minute measurement intervals but we also study the effects of the scale length for 2, 10, 20, and 40 minute intervals. Our analysis of the full set of intervals has shown that:
1. The MV direction of the magnetic field and velocity fluctuations is well defined in $\approx 65 \%$ of cases regardless of scale (Table 1).

2. About $90 \%$ of the magnetic field variations exhibit almost linear polarization, 9\% have elliptical polarization, and the remaining cases $(<1 \%)$ are almost isotropic (Figure 1, left).

3. Velocity fluctuations tend to be more isotropic; the ratios of linear, elliptic, and isotropic variations are approximately $77 \%, 19 \%$, and $4 \%$, respectively (Figure 1, right).

4. Distributions of $\Theta_{B-B 0}$ and $\Theta_{V-B 0}$ angles suggest that the MV direction of the magnetic fluctuations is predominantly aligned with the background magnetic field, but this trend is not so distinct for the velocity variations, especially in high- $\beta$ conditions (Figure 2, left panels).

5. The distribution of the $\Theta_{B-V 0}$ angle is random for $\beta>0.5$ and tends to peak at $45^{\circ}\left(135^{\circ}\right)$ for $\beta<0.1$ (Figure 2, top right panel).

6. The distribution of the $\Theta_{V-V 0}$ angle is random regardless of $\beta$ (Figure 2, bottom right panel).

These results (Figures 1 and 2) show that the background velocity direction (solar wind expansion) does not organize the anisotropy of fluctuations at the considered scale corresponding to 20 minutes in the spacecraft frame, whereas the anisotropy is controlled by the mean background magnetic field. For this reason, we carry out a systematic study of the relation between the MV and mean magnetic field directions using only the cases where the MV direction is well defined (quadrants 1 and 3 in Figure 1). This study reveals that:

1. The angles between the mean magnetic field, $B 0$, and the MV directions of magnetic field $\left(\Theta_{B-B 0}\right)$ or velocity $\left(\Theta_{V-B 0}\right)$ fluctuations are either close to $0^{\circ}$ or to $90^{\circ}$ in $\approx 63 \%$ of cases (Table 1).

2. The most frequent combination is $0-0^{\circ}$, i.e., both $\mathrm{MV}$ directions are aligned with the mean magnetic field (red areas or red points in Figures 1, 5 and 8); these fluctuations have properties consistent with Alfvén waves.

3. The combination of $90-90^{\circ}$ (blue areas or blue points in Figures 1, 5 and 8) exists in 23\% of the 40 minute intervals (Table 1); these variations are probably attributed to slow-mode-like variations with a wavevector quasiperpendicular to the background magnetic field. 
4. This interpretation is supported by their compressive natureaverage $\mathrm{d}|B| /|B 0|$ is by a factor of 5 greater than that in intervals occupied by Alfvénic variations in experiment (Figure 6) and this factor is as large as 10 in simulations.

5. The portion of intervals dominated by slow-mode-like fluctuations decreases with scale and is almost missing in the analyzed 2 minute intervals; this decrease leads to an increase in the relative proportion of Alfvénic fluctuations (Figure 8, Table 1).

6. The intervals occupied with the Alfvénic fluctuations dominate at low $\beta \quad(<0.1)$, whereas slow-mode-like fluctuations are more frequent at large $\beta(>0.5)$ (Figure 5).

7. There is a significant portion of cases with $\Theta_{V-B 0} \approx 90^{\circ}$ and $\Theta_{B-B 0}$ close to $0^{\circ}$ (Figure 5 and 8 ). These intervals are probably dominated by slow modes propagating along the magnetic field but their relative abundance decreases with increasing scale from $23 \%$ for 2 minute intervals to $16 \%$ for 40 minute intervals, i.e., they behave like Alfvénic fluctuations in this respect (Table 1).

8. The above proportions are independent of the application of the mean magnetic field computed on the considered scale (local field) or a "global" mean magnetic field (computed on the 40 minute scale with the analyzed interval in the center).

9. The proportion between Alfvénic and slow-mode-like fluctuations at a given scale is controlled by $\beta$ whereas the solar wind speed plays a minor role (Figure 7).

10. Our MHD simulations support the role of plasma $\beta$ as a control parameter of the proportion/distribution of Alfvénic and slow-mode-like fluctuations.

\section{Concluding Remarks}

We perform a systematic statistical study of turbulent fluctuations in the solar wind. We use the MVA technique to identify different components of magnetic field and velocity fluctuations and their mutual relations. Our analysis indicates a dominance of Alfvénic fluctuations with an MV direction aligned with the background magnetic field that is even more pronounced on shorter scales.

We briefly summarize that the proportions between the different types of magnetic field fluctuations (Alfvénic vs slowmode-like) given in the Summary section are consistent with published results (e.g., Bruno \& Bavassano 1993; Klein et al. 1993; Tu \& Marsch 1995; Ghosh \& Roberts 2012; Klein et al. 2012). However, our numbers have a somewhat different meaning compared to these previous results. Whereas the ratios published so far are related to a mixture of modes existing simultaneously at a particular time and location, our analysis shows a proportion among times dominated by particular types of fluctuations. The data were collected over $7 \mathrm{yr}$ of the Spektr-R operation and sampled by periods of the spacecraft being in the solar wind. The intervals occupied predominantly by a particular type of fluctuation are distributed randomly in our data set. An application of Taylor's hypothesis allows us to conclude that the sampled part of interplanetary space (low latitudes, around $1 \mathrm{au}$ ) is populated by Alfvénic fluctuations with randomly distributed spots of slow-mode-like variations that occupy a scale-dependent percentage of this space. A possible association of these spots with distinct solar wind structures (interplanetary shocks, interplanetary coronal mass ejections, stream interaction regions, etc.) will be a subject of a follow-up study.

The authors acknowledge the Wind team for the magnetic field data available via http://cdaweb.gsfc.nasa.gov/; the BMSW data (Spektr-R project) are available via http:// aurora.troja.mff.cuni.cz/spektr-r/project/. The work of the Czech authors was supported by the Czech Science Foundation under Contract 19-18993S and Charles University grant Agency under No. 264220. The simulation runs were granted by access to the HPC resources of CINES and IDRIS under the allocations 2018-A0050407683 made by GENCI. D.V. is supported by STFC Ernest Rutherford Fellowship ST/ P003826/1 and STFC Consolidated grant ST/S000240/1.

Facilities: Spektr-R, Wind.

\section{ORCID iDs}

Jana Šafránková (10 https://orcid.org/0000-0003-4178-5206 Zdeněk Němeček (i) https://orcid.org/0000-0002-8160-3051 Victor Montagud-Camps (ib https://orcid.org/0000-00027848-9200

Daniel Verscharen (ib https://orcid.org/0000-0002-0497-1096 Andrea Verdini (iD https://orcid.org/0000-0003-4380-4837

\section{References}

Adhikari, L., Zank, G. P., Telloni, D., et al. 2017, ApJ, 851, 117

Bavassano, B., Dobrowolny, M., Mariani, F., \& Ness, N. F. 1982, JGR, 87,3617

Belcher, J. W., \& Davis, L., Jr. 1971, JGR, 76, 3534

Belcher, J. W., \& Solodyna, C. V. 1975, JGR, 80, 181

Bieber, J. W., Wanner, W., \& Matthaeus, W. H. 1996, JGR, 101, 2511

Bruno, R., \& Bavassano, B. 1993, P\&SS, 41, 677

Bruno, R., \& Carbone, V. 2013, LRSP, 10, 2

Burlaga, L. F., \& Turner, J. M. 1976, JGR, 81, 73

Chen, Y., Hu, Q., Zhao, L.-L., et al. 2020, ApJ, 903, 76

Dasso, S., Milano, L. J., Matthaeus, W. H., \& Smith, C. W. 2005, ApJL, 635, L181

Ghosh, S., \& Roberts, D. A. 2012, in AIP Conf. Proc. 1436, Physics of the Heliosphere: A 10 Year Retrospective, ed. J. Heerikhuisen et al. (Melville, NY: AIP), 302

Grappin, R., Velli, M., \& Mangeney, A. 1993, PhRvL, 70, 2190

Hamilton, K., Smith, C. W., Vasquez, B. J., \& Leamon, R. J. 2008, JGR, 113 , A01106

He, J., Tu, C., Marsch, E., \& Yao, S. 2012, ApJL, 745, L8

Howes, G. G., Bale, S. D., Klein, K. G., et al. 2012, ApJL, 753, L19

Klein, K. G., Howes, G. G., TenBarge, et al. 2012, ApJ, 755, 159

Klein, K. G., Howes, G. G., TenBarge, J. M., \& Podesta, J. J. 2014, ApJ, 785,138

Klein, L., Bruno, R., Bavassano, B., \& Rosenbauer, H. 1993, JGR, 98, 7837

Lepping, R. P., Acuna, M. H., Burlaga, L. F., et al. 1995, SSRv, 71, 207

Marsch, E., \& Tu, C.-Y. 1990, JGR, 95, 11945

Matthaeus, W. H., Goldstein, M. L., \& Roberts, D. A. 1990, JGR, 95, 20673

Montagud-Camps, V., Grappin, R., \& Verdini, A. 2018, ApJ, 853, 153

Montagud-Camps, V., Grappin, R., \& Verdini, A. 2020, ApJ, 902, 34

Oughton, S., Matthaeus, W. H., Wan, M., \& Osman, K. T. 2015, Philos. Trans. R. Soc. A-Math. Phys. Eng. Sci., 373, 20140152

Podesta, J. J., \& Gary, S. P. 2011, ApJ, 734, 15

Saur, J., \& Bieber, J. 1999, JGR, 104, 9975

Sonnerup, B. U. O., \& Cahill, L. J. 1967, JGR, 72, 171

Tu, C.-Y., \& Marsch, E. 1994, JGR, 99, 21

Tu, C.-Y., \& Marsch, E. 1995, SSRv, 73, 1

Tu, C.-Y., Roberts, D. A., \& Goldstein, M. L. 1989, JGR, 94, 13575

Verdini, A., \& Grappin, R. 2016, ApJ, 831, 179

Verscharen, D., Klein, K. G., Bennett, A., \& Maruca, B. A. 2019, LRSP, 16, 5

Šafránková, J., Němeček, Z., Němec, F., et al. 2015, ApJ, 803, 107

Šafránková, J., Němeček, Z., Němec, F., et al. 2016, ApJ, 825, 121 
Šafránková, J., Němeček, Z., Němec, F., et al. 2019, ApJ, 870, 40

Šafránková, J., Němeček, Z., Přech, L., et al. 2013, SSRv, 175, 165

Šafránková, J., Němeček, Dušík, Š., Přech, L., Sibeck, D. G., \& Borodkova, N. N. 2002, AnGeo, 20, 301

Zank, G. P., Adhikari, L., Hunana, P., et al. 2017, ApJ, 835, 147

Zank, G. P., \& Matthaeus, W. H. 1992, JGR, 97, 17189

Zank, G. P., \& Matthaeus, W. H. 1993, PhFl, 5, 257
Zank, G. P., Nakanotani, M., Zhao, L.-L., Adhikari, L., \& Telloni, D. 2020, ApJ, 900, 115

Zhao, L.-L., Adhikari, L., Zank, G. P., Hu, Q., \& Feng, X. S. 2018a, ApJ, 856,94

Zhao, L.-L., Zank, G. P., Adhikari, L., et al. 2020a, ApJ, 898, 113

Zhao, L.-L., Zank, G. P., Adhikari, L., et al. 2020b, ApJS, 246, 26

Zhao, L.-L., Zank, G. P., Khabarova, O., et al. 2018b, ApJL, 864, L34 